\title{
Clinical practice guidelines for cancer care: what are they for?
}

\author{
Paula Manchon Walsh · Josep M. Borràs
}

$\mathrm{C}$ linical practice guidelines (CPGs) have been the subject of an extensive effort from many clinicians and methodologists during recent decades. Many organisations such as scientific societies and health services have produced CPGs with heterogeneous results, partially due to the divergent demands placed on them by the organisations that lead the development of the CPGs in each case [1]. In this issue, a new addition to the clinical guidelines literature on the most frequent tumours is published [2] and developed in the framework of the Catalan Cancer Strategy [3], which is a relevant opportunity to briefly review their potential advantages and limitations in the context of our health system.

The standard definition of CPGs is 'systematically developed statements to assist practitioners and patient decisions about appropriate health care for specific circumstances' [4]. Classical accounts of their potential benefits when appropriately developed include the improved clinical outcomes and clinical decisions due to the promotion of interventions with demonstrated efficacy, reducing the well known variability in clinical practice, defining standards of quality of cancer diagnosis and therapy, and offering the possibility to assess their clinical application, as well as helping to recognise gaps in knowledge that require research. On the other hand, the limitations of CPGs are principally those derived from the lack of scientific evidence that could be misinterpreted; as a consequence there is a risk of developing CPGs strongly dependent on the clinical expertise and opinions of the members of the development team. Additional problems in the development of CPGs are related to the limited perspective regarding evidence when only a component of a complex therapy is the aim of the

\author{
J.M. Borràs · P. Manchon Walsh ( $₫$ - \\ Cancer Strategy \\ Department of Health \\ University of Barcelona \\ Av. Gran Vía s/n, km 2,7 \\ 08907 L'Hospitalet de Llobregat, Barcelona, Spain \\ e-mail: pmanchon@iconcologia.net \\ J.M. Borràs \\ Department of Clinical Sciences \\ IDIBELL, University of Barcelona \\ Barcelona, Spain
}

CPGs, a frequent situation when a scientific society is the promoter of the guideline; and last, but not least, the management of the potential conflicts of interest among those involved in the CPGs process [5]. Many of these problems are dealt with in numerous methodological guidelines that have been developed in recent years in order to set the standards of methodology and assess CPGs. Among them we think that it is necessary to mention here the effort carried out by several Spanish agencies for health technology assessment that have started the project Guiasalud (www. guiasalud.es), which publishes on its website all the CPGs that have followed the standards defined, also available there. It is worth mentioning here that this is a project approved by the Ministry of Health and the Autonomous Communities.

Several initiatives are specifically aimed at reviewing the evidence and assessing the quality of CPGs in cancer care. The European project CoCanCPG (Coordination of Cancer Clinical Practice Guidelines in Europe) involves Catalan and Andalusian agencies for technology assessment. CoCanCPG has highlighted the differences and similarities among the CPGs produced across Europe, the need to avoid the repetition of efforts made and the possibility to adapt high quality guidelines to the specificities of the health service considered [6]. Several cancer plans have initiated the development of CPGs for different tumours and the recently approved Spanish strategy has included an objective to have a unique CPG for each tumour site based on all those previously published, in cooperation with the scientific societies, obviously with an assessment of their quality. It is assumed that, if our aim is the equity of cancer care across Spain, patients should know what they should expect from the health service, independently of their place of residence [7]. However, this project is not easy to undertake and we need to bear in mind what the practical problems might be.

Everybody that has been involved in one way or another in the development of CPGs knows that there are three key practical issues that should be dealt with: how to assess the quality of the evidence and what to do when the evidence is weak, how to periodically update the CPGs in order to make them consistent with the progress of research and clinical practice, and how to assess the implementation of the recommendations of the CPGs [8]. 


\section{How have the CPGs published here coped with these problems?}

First of all, the whole process of care has been considered, not focusing only on one therapeutic strategy, and a multidisciplinary team has assessed the evidence. This approach has advantages because it fosters a consensus in a key issue among clinical practitioners with the support of methodologists. A further step made here has been that, in the absence of evidence, expert consensus has been recommended. This controversial option was based on the known fact that clinicians in these cases propose a therapy: we wanted to include what is carried out, if it seems reasonable to the whole team, and propose participation in clinical trials if available.

Second, the CPGs published here are the updated version of the first CPGs published 5 years ago. Now there is a small committee that continuously reviews the evidence published and can propose changes to the CPG if needed. In a field as innovative as cancer care, all the effort invested in the development of a CPG is worthless if not updated periodically (less than every 2 years).

Finally, an assessment of the quality and outcomes of cancer diagnosis and care in cancer of the rectum, using as a reference the corresponding CPG, has been recently completed and the results will soon be available. We believe that the mentioned 'Rectal cancer audit' is the best example of a commitment to assess the implementation of the recommendations included in a CPG. Therefore we aim at repeating the same process every year on behalf of one updated CPG on a different tumour site.

This approach of building a process of CPG with the involvement of a multidisciplinary and extensive team of clinicians has proved to be successful in other experiences. In a French region, the lack of agreement with the recommendations included in the consensus breast cancer CPG ranged from $12.6 \%$ to $25.2 \%$, the age (older) and prognosis (good) of the patient being the main factors associated to lack of compliance [9]. Also, there is evidence of the impact of the CPG on changing clinician behaviour in the expected direction, although there is no single strategy clearly better than any other in such an implementation process $[10,11]$. In summary, there is evidence supporting that the opportunities offered by CPGs improve clinical practice and those published here are an example of this approach.

An additional advantage of a CPG when a summary of information for patients is added is that the patients are given the opportunity to know what care they should expect. This point emphasises support for improving equity of cancer care across providers.

To summarise, CPGs could be a tool to reduce the variability in clinical practice and an opportunity to reach agreement among oncologists regarding the best care based on the available evidence, and they could provide a framework to the patients regarding the quality and the therapy they should expect, improving the equity of care. However, without a strong commitment to the assessment of the implementation of the recommendations and the outcomes of the cancer therapy in each hospital, their effectiveness could be reduced to an academic effort. Finally, CPG recommendations should be applied with quality in clinical practice. Probably the best we can do for future patients is to focus our efforts on research but for today's patients it is to apply what we know is effective with quality and evaluate our results so that we know how we are doing and make improvements where necessary [12].

Acknowledgements This paper was partially funded by the Instituto Carlos III (RTICC. RD 06/0020/0089).

\section{References}

1. Pentheroudakis G, Stahel R, Hansen H, Pavlidis N (2008) Heterogeneity in cancer guidelines: should we eradicate or tolerate? Ann Oncol 19:2067-2078

2. Manchon $\mathrm{P}$, Borràs $\mathrm{JM}$, Ferro $\mathrm{T}$, Espinàs JA (2009) Lung Cancer OncoGuia. Clin Trans Oncol 11:805-824

3. Pla director d'oncologia 2008-2010. Barcelona: Departament de salut, 2009. Available from: http://www.gencat.cat/salut/depsalut/html/ca/ dir2894/index.html

4. Field MJ, Lohr KN (eds) (1990) Clinical practice guidelines: directions for a new program. National Academy Press, Washington, DC
5. Wolf SH, Grol R, Hutchinson A et al (1999) Potential benefits, limitations and harms of clinical practice. BMJ 318:527-530

6. Fervers B, Remy-Stockinger M, Mazeau-Woynar $\mathrm{V}$ et al (2008) CoCanCPG. Coordination of cancer clinical practice in Europe. Tumori 94:154159

7. Murad MH, Montori VM, Guyatt GH (2008) Incorporating patient preferences in evidence-based medicine. JAMA 300:2483-2484

8. National Institute for Health and Clinical Excellence (2009) The guidelines manual. National Institute for Health and Clinical Excellence, London. Available from: www.nice.org.uk

9. Eisinger F, Ronda I, Puig B et al (2006) Breast cancer guidelines: physicians, intentions, behaviours. Int J Cancer 120:1136-1140

10. Ray-Coquard I, Phillip T, de Laroche G et al (2005) Persistence of medical change at implementation of clinical guidelines on medical practice: a controlled study in a cancer network. J Clin Oncol 23:4414-4423

11. Grol R, Grimshaw J (2003) From best evidence to best practice: effective implementation of change in patients care. Lancet 362:1225-1230

12. Woolf SH, Jonson RE (2005) The break even point: when medical advances are less important than improving the fidelity with which they are delivered. Ann Family Med 3:545-552 